\title{
Comment on the Research Progress of the Impact of Organizational Citizenship Pressure
}

\author{
Jie Chen, Liangtie Dai \\ Management School, Jinan University, Guangzhou, China \\ Email: 15521089599@163.com
}

How to cite this paper: Chen, J., \& Dai, L. T. (2018). Comment on the Research Progress of the Impact of Organizational Citizenship Pressure. Psychology, 9, 985-996. https://doi.org/10.4236/psych.2018.95062

Received: March 7, 2018

Accepted: May 11, 2018

Published: May 17, 2018

Copyright $\odot 2018$ by authors and Scientific Research Publishing Inc. This work is licensed under the Creative Commons Attribution International License (CC BY 4.0).

http://creativecommons.org/licenses/by/4.0/

(c) (i) Open Access

\begin{abstract}
Previous studies have generally focused on the OCB, in which employees voluntarily engage, and while because OCB is often informally encouraged or rewarded, employees may experience the pressure to become "good Staff", that is, Organizational Citizen Pressure. This article systematically explores the formation of the concept of Organizational Citizen Pressure, the possible positive and negative impacts it may have, and related research theory. The paper also looks into the future research direction of Organizational Citizen Pressure. Due to the double-edged sword effect of Organizational Citizen Pressure, a full understanding of it will be of great guidance meaning to organizational management theory and practice.
\end{abstract}

\section{Keywords}

Organizational Citizen Pressure, Organizational Citizenship Behavior (OCB), Double-Edged Sword Effect

\section{Introduction}

Smith, Organ and Near (1983) proposed the concept of organizational citizenship behavior (OCB) and defined it as an employee's spontaneous, indirectly and explicitly incorporated into the rewards and punishments of the organization, and this behavior can promote the effectiveness of the organization (Organ, 1988). For more than 30 years, the concept of OCB has focused on the positive impact of OCB. For example, research shows that employees who perform OCB have more potential benefits over other employees, including gaining help from others, high job satisfaction, and high performance appraisal results (Lyons \& Scott, 2012). Until the early 21st century, with the rapid economic development, managers began to pay attention to and encourage employees to engage in OCB 
(Zhao \& Jiang, 2017a). And even more and more companies typically rewarded employees who perform OCB (Podsakoff, Podsakoff, MacKenzie, Maynes, \& Spoelma, 2014). As managers frequently emphasize and encourage OCB, the researchers found that there is also a phenomenon of "job creep" in enterprises, that is, the gradually expanding of employees' roles and responsibilities, and more and more organizational citizenship behavior which is obviously extra-role behavior is also increasingly being seen as role behavior (Van Dyne \& Ellis; 2004). When starting a new job, employees' job responsibilities are clearer. Any kind of OCB may be considered as extra-role behavior. As employees spend more time with $\mathrm{OCB}$, the corresponding organizational citizenship behavior is increasingly seen as an obligation within the role, and as time goes by, the boundaries between roles and extra-role become increasingly blurred (Morrison 1994). In this professional context, employees are increasingly experiencing the pressure to become a "good employee", which is organizational citizenship pressure (Bolino, William, Gilstrap, \& Suazo, 2010). According to a survey conducted in People's Daily Online-Global Times in 2006, China has become one of the countries with long working hours in the world. Overtime has become Chinese workplace culture. Many employees take the initiative to work overtime for fear of losing their jobs. According to the data, over 600,000 people have died each year from work in China. The concept of organizational citizenship pressure was put forward relatively late, and the empirical research in related fields is relatively scarce. However, take overtime working as an example, most employees not to really willingly to but pressured to work overtime. The negative impact of organizational citizenship pressure, like the pressure to work overtime, has caused widespread social concern before the concept of organizational citizenship pressure was put forward. Therefore, in the ten years after Bolino et al. (2010) put forward the concept of organizational citizenship pressure, organizational citizenship pressure has drawn the widespread attention of theorists and practitioners.

Jamal's study (1999) shows that proper levels of stress can improve employee morale and motivate employees' motivation and enthusiasm for their work, and when stress exceeds individual-controllable levels, they can lead to physical (e.g., increased blood pressure, Metabolic disorders, etc.) and psychological (such as anxiety, depression) discomfort, thereby affecting the performance of staff work. It is still unknown that, as a kind of pressure, whether the organizational citizenship pressure is just like the general pressure, having the "inverted U-shaped" curve effect. This paper systematically sorts out the origin of the concept of organizational citizenship stress and its theoretical and empirical research results on both positive and negative impact on enterprises and individuals; and summarizes its regulatory factors that affect the results. At the same time, this paper evaluates and the existing research on the organizational citizenship pressure in order to provide meaningful reference and inspiration for the development of organizational citizen pressure research in the future. 


\section{The Formation of the Concept of Organizational Citizenship Pressure}

Organizational Citizenship pressure is a concept derived from OCB and refers to the pressure perceived by employees when they are unwillingly to perform OCB. Numerous studies at the end of the 20th century showed that OCB has a positive impact on both organizations and individuals in many aspects. Organizational managers also encourage or even reward employees for performing OCB, resulting in gradual job creep and increasing employee pressure of being forced to perform organizational citizenship behavior (OCB).

\subsection{Organizational Citizenship Behavior (OCB)}

There is a clear difference in the behavior of employees in the organization; from the "minimalists", who is only willing to engage in behavior which is enough to maintain the membership of the organization, to the organization "good citizen", who is willing to do more extra role behavior which is expected, can promote the effective operation of the organization or is beneficial to others. Employees' spontaneous, not evaluated by performance assessment system and welcomed by the organization behavior is called organizational citizenship behavior (OCB) or good soldier syndrome (Organ, 1988). In the early days, researchers have mostly focused on exploring the positive impact of OCB on individuals and organizations. For individuals, according to social exchange theory, employees with higher levels of helpfulness are more likely to be helped by others (Lyons et al., 2012); In addition, job satisfaction, job performance, and the willingness to resign have also been shown to be significantly positively correlated with OCB (Organ \& Lingl, 1994). Organizational managers prefer those subordinate who would go beyond their duty to help or supervise their colleagues, actively participate in organizing activities, make recommendations and be concerned about the development of the organization (Whiting et al., 2008). Although OCB has not be consider as one of the standard of performance appraisal, a large number of studies still show that employees' OCB can positively influence the result of superior appraisal and even affect the possibility of promotion (Podsakoff et al., 2008). For organizations, when OCB become a common phenomenon in a organization, the organization would be more attractive to employees (Organ, Podsakoff, \& MacKenzie, 2006). Organizations also accumulate their own social capital due to their possession of "good citizens", so as to improve the operational efficiency of organization and enhance their competitive advantages (Organ et al., 2006). Therefore, the early managers also fully encouraged, promoted and nurtured the OCB of their employees in their management practices.

\subsection{Job Creep}

However, with the change of corporate culture and market competition environment, organizational citizenship behaviors (OCB) are losing their spontanei- 
ty. More and more business managers will "request" employees to do extra work, such as prolonged overtime working, frequently bordering employees during their work vacations. Theoretical studies (Salamon \& Deutsch, 2006) and empirical studies (Bolino et al., 2010) show that in more and more organizational contexts, the employees' OCB is not really voluntary or even forced. When employees engage in organizational citizenship behaviors (OCB), their behaviors are gradually regarded as normalized and routine organizational behaviors; as a result, employees need to be engaged in more extra-role behaviors so that they could be considered as good citizens of the organization (Bolino \& Turnley, 2003). Van Dyne et al. (2004) proposed the concept of job creep to explain this phenomenon. Job creep reflects the informal expansion of job responsibilities. As superiors and co-workers gradually identify employee spontaneous OCB as role behavior, the boundaries between role behavior and extra-role behavior are increasingly difficult to distinguish. Employees' spontaneous OCBs are no longer seen as extra work behavior beyond their job requirements but as a kind of job requirements.

\subsection{Organizational Citizenship Pressure}

Simultaneously with the notion of job creep being proposed, Schaufeli and Bakker (2004) put forward the notion of job demands which refers to the continuous physiological or psychological (e.g., cognitive or emotional) pay or needed to maintain a job and the related physical or psychological costs. Schaufeli et al. (2004) also emphasized that one of the typical symptom of job demands is the pressure experienced by employees who want to meet their job demands.

Bolino et al. (2010) proposed the notion of "citizenship pressure" based on the concepts of job creep and job demands, defining organizational citizenship pressure as a specific job demands; the pressure employees perceived when they are forced into performing organizational citizenship behavior. Relatively confusing concepts are individual's subjective OCB norms, the culture of citizenship and the citizenship climate (Bolino et al., 2010). Ehrhart and Naumann (2004) proposed the notion of individual's subjective OCB norms, refers to the extent to which individuals think their co-workers expect themselves to perform OCB , or the extent to which OCB within the organization is considered desirable or reasonable. Culture of citizenship refers to the organizational culture which encourages OCB by treating people fairly, offering interesting or satisfying jobs, or meeting diverse employee needs (Chen, 2008). Citizenship climate refers to the organizational climate in which employees are generally accredited and tend to spontaneously engage in an organizational climate of high levels of OCB (Tepper, Duffy, Hoobler, \& Ensley, 2004).

According to exchange theory, Lazarus (1966) suggests that stress is the psychological and physiological reaction that occurs when an individual perceives an imbalance between the needs imposed on him and the individual's ability to cope with the needs. As a kind of stress, organizational citizenship pressure is neither a perception nor an organizational culture or organizational climate. 


\section{The Impact of Organizational Citizenship Pressure}

At present, there are two different views on the outcomes of organizational citizenship pressure. For example, one thinks that the organizational citizenship pressure can improve the organizational citizenship behavior of employees; the other indicates that organizational citizenship pressure can reduce the altruism and voluntary of organizational citizenship behavior and in turn reduce the employees' organizational citizenship behavior. This section summarizes the research on the positive and negative impact of organizational citizen pressure.

\subsection{The Positive Impact of Organizing Civil Pressure}

At present, there are relatively few empirical and theoretical studies on the positive impact of organizational citizenship pressure. Some studies have shown that organizational citizenship pressure can promote the employee OCB. Case studies of McAllister, Kamdar, Morrison, and Turban (2007), using interpersonal helping and taking charge as examples, explored the relationship between Perceived Role Breadth and Organizational Citizenship Behavior (OCB) in OCB. Perceived role breadth of organizational citizenship refers to the extent to which employees consider a particular OCB as their own role behavior. The results show that employees who consider helping behavior and dedication as his role behavior have a higher level of helpfulness or a higher level of dedication. Based on this research, Bolino et al. (2010) put forward hypotheses based on the challenge stress-hindrance stressor framework (LePine, Podsakoff, \& LePine, 2005) and social exchange theory that the level of citizenship pressure has a positive effect on OCB and is verified through empirical research across time.

Zhao Hongdan and Jiang Wei (2017b) argue that lateral advice (ie, objections to colleagues aimed at improving the organization's work efficiency) helps employees mitigate the stress and insecurity stemming from citizenship pressure; therefor, they hypothesized that organizational citizenship pressures in the workplace can positively influence the employee's lateral voice behavior and verify the results through empirical research.

In addition, according to role accumulation theory, when an individual feels that he/she is obligated to perform tasks other than job responsibilities, the experience of stress helps to improve employee awareness and concentration of work, enhance employee motivation and emotional and Physiological vitality. At the same time, as a result of positive spillover, the effectiveness of a character will promote the performance of another character. Cates, Mathis and Randle (2010) show through empirical research that organizational citizenship pressure can increase the work input of employees and further enhance the work-to-family enrichment of employees.

\subsection{The Negative Impact of Organizational Citizenship Pressure}

For individual employees, on the one hand, studies have shown that organizational citizenship pressure increases the overall work pressure of employees, the 
willingness to resign, the decline in well-being, and the work and leisure conflicts (Ilies, Aw, \& Lim, 2016). In the meantime, when the level of organizational citizenship is high, it will in turn increase the employee's fatigue, that is, the sense of tiredness and fatigue experienced by employees when engage in OCB (Zhao et al., 2017b); thereby, employees would further reduce OCB (Bolino \& Klotz, 2015). Yam, Klotz, He and Reynolds (2017) argued that when an employee feels forced to engage in OCB due to external forces, the employee psychologically believes they have surpassed their own job responsibilities and considers themselves mentally and psychologically acquired eligibility for interpersonal or organizational deviant behavior. According to moral licensing, when organizational citizenship pressure is high, those employees who would perform counterproductive behavior so as to maintain a moral balance would accordingly reduce their pressure(contrasting employees who refuse to engage in counterproductive behavior) (Klotz \& Bolino, 2013). As a result, organizational citizenship pressure will not only reduce employee OCB but may even increase employee counterproductive behavior.

On the other hand, organizational citizenship pressures also have a negative impact on employees' family lives; employees who feel higher levels of organizational citizenship in their work also experience similar pressures at home, such as the pressure to become a good husband or a good father (Liu, Zhao, \& Sheard, 2017). Due to the role conflict, higher levels of organizational citizenship pressure often increase the conflict between work and family (Bolino et al., 2010).

For the organization, organizational citizenship pressure will negatively affect the employee's upward voice behavior (Zhao et al., 2017b), which may indirectly affect organizational performance. At the same time, employees who experience a higher level of organizational citizenship pressure may find the organization's attractiveness declining and may complain about the organization or have the willingness to resign (Ilies et al., 2016). As a result, higher organizational citizenship pressures may result in higher turnover rates for the organization or difficult to keep good employees, thereby reducing the competitiveness of the organization.

\section{The Analysis of the "Double-Edged Sword" Effect of Organizational Citizenship Pressure}

Based on the above review of the study on the impact of organizational citizenship pressure, we can find that it has both positive and negative aspects, and even there are inconsistencies in the conclusions about the same outcome variable. For example, whether organizational citizenship pressures promote OCB or reduce employee OCB; whether organizational citizenship pressure leads to work-family conflicts or to work-family gains. This shows that the impact of organizational citizenship pressure has complexity; and so far, the predictive validity is not obvious. As for the double-edged sword effect of the organizational citizenship pressure, the explanations put forward by the researcher are mainly in 
two aspects: there are individual differences, and at the same time, they depend to a certain extent on the organizational situation. Representative theories include Job Demands-Resources Model, Challenge Stressor-Hindrance Stressor Framework, Social Exchange Theory, Impression and Management Theory.

\subsection{Job Demands-Resources Model}

According to the job demands-resource model (Schaufeli et al., 2005), each job has its own specific characteristics related to job stress and job burnout. These characteristics can be divided into job demands and job resources. When employees are in an organizational context where job demands and resources are high, employees perceive high job demnads as "challenges" to themselves and show positive working attitude and work harder. When the employees are in Organizational context with higher job demands, lower resources, the enthusiasm of employees will suffer; and they are likely to show slack off.

Self-efficacy is a type of work resource; a worker with a high sense of self-efficacy (rich work resources) sees organizational citizenship pressure (job demands) as a challenge to themselves, stimulating morale and showing a high level of OCB. Employees with low self-efficacy (lack of work resources) would easily get organizational citizenship fatigued, when they feel organizational citizenship pressure and thus showing a lower level of OCB (Zhao et al., 2017b). Employees could save their work resources by establishing positive, supportive exchange relationships with their leaders, which can also reduce their sense of organizational citizenship fatigue and thereby promote employee OCB (Bolino et al., 2015). According to Job Demands-Resources Model, there are individual differences in the stock of employees' work resources (e.g., self-efficacy); it's also affected by the different organizational context (e.g., the exchange leadership).

\subsection{The Challenge Stressor-Hindrance Stressor Framework}

According to the Challenge Stressor-Hindrance Stressor Framework, stressors can be divided into challenge stressors and hindrance stressors. The expectations theory can be used to better understand how Challenge Stressor-Hindrance Stressor Framework influence employees' motivation and behavior (LePine et al., 2004). Firstly, whether the stressor-related demand can be met through hard work, and secondly, whether meeting the demands will brought employees some value or effectiveness. The Challenge Stressor-Hindrance Stressor Framework are often associated with high motivation because people believe that challenging stressors can be relieved after hard-working and that individuals can feel a sense of fulfillment and value when those stressors are relieved. For example, organizational citizenship pressure is a kind of challenge stressor employees can relieve it by engaging in a higher level of OCB (Bolino et al., 2010). And after meeting their needs, employees feel the pride of being a good employee. Hindrance stressor are usually associated with low motivation because one believes that the stressor's needs can not be met by paying more, or, even if that need is 
met, there is no sense of accomplishment or value for the individual sense. For example, role conflict is a kind of hindrance stressor; it is generally believed that when there is a conflict between multiple roles, one can not strive to meet the needs of multiple roles simultaneously. Organizational citizenship pressure is often accompanied by role conflict (Bolino et al., 2010). Role conflict, as a kind of hindrance stressor, would reduce employee motivation to engage in OCB and reduce OCB.

According to the Challenge Stressor-Hindrance Stressor Framework, organizational citizenship pressure as a kind of challenging stress can motivate employees to engage in more OCBs; besides, it may also reduce the motivation of employees to engage in OCB by causing role conflict.

\subsection{The Social Exchange Theory}

Social exchange theory emphasizes the relationship that employees form at work. Social exchange theory is often used to describe two types of interpersonal relationships; economic exchange relationships and social exchange relationships. Economic exchange relationship generally refers to the short-term, relatively specific, often economic, exchange of benefits (e.g., payment). The social exchange relationship is different from the economic exchange. The social exchange relationship takes the social emotional benefits as the exchange content. It includes close personal relationships and open obligations (e.g., respect and care). In organizations with high social exchange relationships, employees have a higher level of OCB and a lower level of willingness to resign (Cropanzano, Rupp, \& Byrne, 2003).

Numerous studies have shown that employees are encouraged to establish positive and supportive exchange relationships with their superiors and perform more OCB when they are given interesting or desirable tasks, or when they feel that they are fairly treated, or when they feel supported or encouraged by their superiors. Conversely, employees who experience a lack of organizational support and a lack of exchange of team members will likely experience a higher degree of organizational citizenship fatigue, thus gradually reducing their OCB. According to the Social exchange theory, when employees feel pressure to engage OCB, employees who have higher satisfaction with their work (compared with staff who are disgusted with work) show higher level of OCB (OCB) (Bolino et al., 2010). Therefore, according to the Social exchange theory, the complexity of the results of organizational citizenship pressure is influenced by various situational factors.

\subsection{The Impression Management}

Impression management refers to the process by which people try to manage and control their impression on others by their behavior, body and verbal language. Impression management motivation refers to the extent to which people want to control others' perceptions of themselves. There are many similarities 
between impression management behavior and OCB. For example, helping one's own leadership, that is, belong to the impression management bahavior, but also to the OCB. Schnake (1991) pointed out that before further understanding of the motivation behind the behavior, impression management behavior is often misunderstood as OCB. Employees may also create their "good employees" image in the eyes of their superiors and colleagues by engaging in more OCBs for the motivation of impression management (Bolino, 1999). According to impression management theory, highly self-monitoring employees (who are more aware of their impression on others) are more likely to engage in higher levels of OCB than those who have lower self-monitoring employees. Bolino et al. (2010), through empirical studies, show that impression management regulates the relationship between organizational citizenship pressure and OCB. When organizational citizenship pressure is high, employees with high self-monitoring have a higher level of OCB.

However, employees who engage in OCB for the sake of impression management will reduce their OCB as soon as they are promoted. Employees are also more likely to engage in counterproductive work behavior in the future as they lose personal resources when performing OCB (Bolino et al., 2015).

Thus, the impact of organizational citizenship pressure on OCB is affected by the discrepancy between individual impression management motives. On the other hand, when the employee with high impression management motivation is promoted or rewarded through OCB, they may reduce or even engage in counterproductive work behavior. In other words, there is a mediating effect of impression management on organizational citizenship pressure and $\mathrm{OCB}$, as well as the differentiated effect of intertemporal and organizational context.

\section{Research Prospect}

Firstly, the conceptual definition of organizational citizenship pressure and the choice of dimensions need to be unified. Bolino et al. (2010) put forward the concept of organizational citizenship stress and at the same time, measured employees' organizational citizenship pressure by measuring their stress level when performing OCB. Most of the subsequent empirical studies on organizational citizenship stress (Zhao et al., 2017b) followed the organizational citizenship pressure scale developed by Bolino (2010). However, Bolino's (2010) definition of organizational citizenship pressure has not been widely recognized by the academic community. Whether the scale developed on the basis of this definition can accurately measure the organizational citizenship pressure accurately requires more discussion and attention from relevant researchers in future research.

Secondly, future research can focus on the double-edged mechanism of organizational citizenship pressure. Many of the above studies show that there is both positive and negative impacts of organizational citizenship pressure on employees' OCB and work-family balance (Liu et al., 2017). Whether the relation- 
ship between organizational citizenship pressure and some outcome variables is an inverse U-curve. Before a certain critical point, there is a positive correlation between the variables, beyond a certain critical point, there is a negative correlation between the variables. Suggestions for future research can also start from this aspect to conduct in-depth discussion.

Finally, researchers should also pay attention to the localization of organizational citizen pressure. At present, the study on the organizational citizenship pressure (Zhao et al., 2017a) mostly adopts the general organizational citizenship pressure scale developed by Bonilo (2015), which was developed under the background of the American culture. There is a big difference between American corporate culture and Chinese corporate culture. For example, the Chinese corporate culture emphasizes interpersonal relationships and authority. Therefore, it is suggested that the organizational citizenship pressure should be measured by the Chinese localization scale in order to ensure higher validity.

\section{General Conclusion}

This article mainly introduces the origin of the concept of organizational citizenship pressure and its outcome variables. In a review of the results of organizational citizenship pressure, it was found that organizational citizenship pressure has its double-edged sword effect. Therefore, this article further explains the reasons of its double-edged sword effect from the related theory, including the job demands-resources model, the challenge stressor-hindrance stressor framework, the social exchange theory and the impression management theory. And on the basis of the existing research, it proposes future research directions.

\section{References}

Bolino, M. C. (1999). Citizenship and Impression Management: Good Soldiers or Good Actors? Academy of Management Review, 24, 82-98.

https://doi.org/10.2307/259038

Bolino, M. C., \& Klotz, A. C. (2015). The Paradox of the Unethical Organizational Citizen: The Link between Organizational Citizenship Behavior and Unethical Behavior at Work. Current Opinion in Psychology, 6, 45-49. https://doi.org/10.1016/j.copsyc.2015.03.026

Bolino, M. C., \& Turnley, W. H. (2003). Going the Extra Mile: Cultivating and Managing Employee Citizenship Behavior [and Executive Commentary]. The Academy of Management Executive (1993-2005), 17, 60-73.

Bolino, M. C., Turnley, W. H., Gilstrap, J. B., \& Suazo, M. M. (2010). Citizenship under Pressure: What's A “Good Soldier” To Do? Journal of Organizational Behavior, 31, 835-855. https://doi.org/10.1002/job.635

Chen, H. (2008). Hypothetical and Theoretical System Framework of Cultivation of Organizational Citizenship Behavior and Validation. International Journal of Business and Management, 20, 637-644.

Cropanzano, R., Rupp, D. E., \& Byrne, Z. S. (2003). The Relationship of Emotional Exhaustion to Work Attitudes, Job Performance, and Organizational Citizenship Behaviors. Journal of Applied Psychology, 88, 160-169.

https://doi.org/10.1037/0021-9010.88.1.160 
Ehrhart, M. G., \& Naumann, S. E. (2004). Organizational Citizenship Behavior in Work Groups: A Group Norms Approach. Journal of Applied Psychology, 89, 960. https://doi.org/10.1037/0021-9010.89.6.960

Ilies, R., Aw, S. S. Y., \& Lim, V. K. G. (2016). A Naturalistic Multilevel Framework for Studying Transient and Chronic Effects of Psychosocial Work Stressors on Employee Health and Well-Being. Applied Psychology, 65, 223-258. https://doi.org/10.1111/apps.12069

Jamal, M. (1999). Job Stress, Type-A Behavior, and Well-Being: A Cross-Cultural Examination. International Journal of Stress Management, 6, 57-67. https://doi.org/10.1023/A:1021962320645

Lazarus, R. S. (1966). Psychological Stress and the Coping Process. New York, NY: McGraw-Hill.

Lepine, J. A., Podsakoff, N. P., \& Lepine, M. A. (2005). A Meta-Analytic Test of the Challenge Stressor-Hindrance Stressor Framework: An Explanation for Inconsistent Relationships among Stressors and Performance. Academy of Management Journal, 48, 764-775. https://doi.org/10.5465/AMJ.2005.18803921

Liu, Y., Zhao, H., \& Sheard, G. (2017). Organizational Citizenship Pressure, Compulsory Citizenship Behavior, and Work-Family Conflict. Social Behavior \& Personality an International Journal, 45, 695-704. https://doi.org/10.2224/sbp.6064

Lyons, B. J., \& Scott, B. A. (2012). Integrating Social Exchange and Affective Explanations for the Receipt of Help and Harm: A Social Network Approach. Organizational Behavior and Human Decision Processes, 117, 66-79.

https://doi.org/10.1016/j.obhdp.2011.10.002

Mcallister, D. J., Kamdar, D., Morrison, E. W., \& Turban, D. B. (2007). Disentangling Role Perceptions: How Perceived Role Breadth, Discretion, Instrumentality, and Efficacy Relate to Helping and Taking Charge. Journal of Applied Psychology, 92, 1200-1211. https://doi.org/10.1037/0021-9010.92.5.1200

Morrison, E. W. (1994). Role Definitions and Organizational Citizenship Behavior: The Importance of the Employee's Perspective. Academy of Management Journal, 37, 1543-1567. https://doi.org/10.2307/256798

Organ, D. W. (1988). Organizational Citizenship Behavior: The Good Soldier Syndrome. Lexington, MA: Lexington Books.

Organ, D. W., \& Lingl, A. (1994). Personality, Satisfaction, and Organizational Citizenship Behavior. Journal of Social Psychology, 135, 339-350. https://doi.org/10.1080/00224545.1995.9713963

Organ, D. W., Podsakoff, P. M., \& MacKenzie, S. B. (2006). Organizational Citizenship Behavior: Its Nature, Antecedents, and Consequences. Personnel Psychology, 59, 484-487. https://doi.org/10.1111/j.1744-6570.2006.00043_9.x

Podsakoff, N. P., Podsakoff, P. M., Mackenzie, S. B., Maynes, T. D., \& Spoelma, T. M. (2014). Consequences of Unit-Level Organizational Citizenship Behaviors: A Review and Recommendations for Future Research. Journal of Organizational Behavior, 35, S87-S119. https://doi.org/10.1002/job.1911

Salamon, S. D., \& Deutsch, Y. (2006). OCB as a Handicap: An Evolutionary Psychological Perspective. Journal of Organizational Behavior, 27, 185-199. https://doi.org/10.1002/job.348

Schaufeli, W. B., \& Bakker, A. B. (2004). Job Demands, Job Resources, and Their Relationship with Burnout and Engagement: A Multi-Sample Study. Journal of Organizational Behavior, 25, 293-315. https://doi.org/10.1002/job.248 
Schnake, M. (1991). Organizational Citizenship: A Review, Proposed Model, and Research Agenda. Human Relations, 44, 735-759. https://doi.org/10.1177/001872679104400706

Smith, C., Organ, D. W., \& Near, J. P. (1983). Organizational Citizenship Behavior: Its Nature and Antecedents. Journal of Applied Psychology, 68, 653-663. https://doi.org/10.1037/0021-9010.68.4.653

Tepper, B. J., Duffy, M. K., Hoobler, J., \& Ensley, M. D. (2004). Moderators of the Relationships between Coworkers' Organizational Citizenship Behavior and Fellow Employees' Attitudes. Journal of Applied Psychology, 89, 455-465. https://doi.org/10.1037/0021-9010.89.3.455

Van Dyne, L., \& Ellis, J. B. (2004). Job Creep: A Reactance Theory Perspective on Organizational Citizenship Behavior as Overfulfillment of Obligations. The Employment Relationship: Examining Psychological and Contextual Perspectives (pp. 181-205). Oxford: Oxford University Press.

Whiting, S. W., Podsakoff, P. M., \& Pierce, J. R. (2008). Effects of Task Performance, Helping, Voice, and Organizational Loyalty on Performance Appraisal Ratings. Journal of Applied Psychology, 93, 125-139. https://doi.org/10.1037/0021-9010.93.1.125

Yam, K. C., Klotz, A., He, W., \& Reynolds, S. (2017). From Good Soldiers to Psychologically Entitled: Examining When and Why Citizenship Behavior Leads to Deviance. Academy of Management Journal, 60, 373-396. https://doi.org/10.5465/amj.2014.0234

Zhao, H., \& Jiang, W. (2017b). The Influences of Citizenship Pressure and Citizenship Fatigue on Organizational Dissent: The Moderating Role of Self-Efficacy. Forecast, 36, 15-20.

Zhao, H., Jiang, W., Management, S. O., et al. (2017a). Citizenship Pressure in the Workplace. Advances in Psychological Science. 